\title{
Quantum aspects of Yukawa model with scalar and axial scalar fields in curved spacetime
}

\author{
Iosif L. Buchbinder ${ }^{2,3, a}$, Andreza Rairis Rodrigues ${ }^{1, b}$, Eduardo Antonio dos Reis ${ }^{1, \mathrm{c}}$, Ilya L. Shapiro ${ }^{1,2,3, \mathrm{~d}}$ \\ ${ }^{1}$ Departamento de Física, ICE, Universidade Federal de Juiz de Fora, Juiz de Fora, MG 36036-330, Brazil \\ ${ }^{2}$ Department of Theoretical Physics, Tomsk State Pedagogical University, Tomsk 634061, Russia \\ ${ }^{3}$ National Research Tomsk State University, Tomsk 634050, Russia
}

Received: 15 October 2019 / Accepted: 30 October 2019/ Published online: 12 December 2019

(C) The Author(s) 2019

\begin{abstract}
We study the Yukawa model with one scalar and one axial scalar fields, coupled to $N$ copies of Dirac fermions, in curved spacetime background. The theory possesses a reach set of coupling constants, including the scalar terms with odd powers of scalar fields in the potential, and constants of non-minimal coupling of the scalar fields to gravity. Using the heat-kernel technique and dimensional regularization, we derive the one-loop divergences, describe the renormalization of the theory under consideration and calculate the full set of beta- and gamma-functions for all coupling constants and fields. As a next step, we construct the renormalized one-loop effective potential of the scalar fields up to the terms linear in scalar curvature. This calculation includes only the contributions from quantum scalar fields, and is performed using covariant cut-off regularization and local momentum representation. Some difficulties of the renormalization group approach to the effective potential in the case under consideration are discussed.
\end{abstract}

\section{Introduction}

The interaction between scalar fields with Dirac spinors through a Yukawa interaction is attracting a special attention in quantum field theory in curved spacetime. In this respect one can mention recent analysis that includes both scalar and a pseudoscalar couplings [1] and more recently with the inclusion of a gauge field [2,3]. In the present work we continue the previous treatment of Yukawa model with sterile scalar discussed in [4] and extend it to the case of the two (ordinary and axial) scalars with a Yukawa coupling to

\footnotetext{
a e-mail: joseph@tspu.edu.ru

b e-mail: andrezarodrigues@ice.ufjf.br

c e-mail: eduardoreis@ice.ufjf.br

d e-mail: shapiro@fisica.ufjf.br
}

fermions and general renormalizable form of self-interaction. Our immediate purpose will be the calculation of divergences in the most economic way, as it was done in the original publication on the renormalization of the Abelian model with Yukawa coupling in curved spacetime with torsion from long ago [5] (see also the book [6]).

Similar consideration of the simpler model with a single scalar field was useful in establishing the constraints on the quantum theory that come from the condition of renormalizability of the Abelian theory with massive Dirac field. The form of the self-interaction potential of a scalar field ensuring the renormalizability of such a theory is an interesting aspect, that was not explored completely in the original work [5]. It was shown and discussed in details in the recent work [4] that the renormalizable scalar with Yukawa interaction includes self-interactions with $o d d$ powers of the scalar fields. These qualitatively new interactions include linear term, the term with a cubic coupling, and also a linear term describing the interaction between scalar field and scalar curvature.

In all examples of renormalizable quantum field theories with scalar fields, which were known until now, it was possible to construct solutions to the renormalization group equation for the effective action which enable to derive the effective potential in the most economic way [7], including in curved spacetime [8] (see also the generalization to other sectors of effective action in [6,9]). In the model with a single sterile scalar one has to extend this nicely working scheme to include odd powers of the scalar field, with this generalization it still works pretty well [4].

The generalization of these considerations to the paritypreserving model with an additional axial scalar field is an interesting and challenging problem. Let us start by stating that this problem makes sense from the viewpoint of physical applications. First of all, there is an important example of an axial scalar, that is an axion. Regardless axion might have different form of coupling to gauge and fermion fields compared 
to an ordinary axial scalar field, it is interesting to explore the renormalization of such a parity-preserving model on a simple example. On the other hand, in the recent years there were indications of the possible violations of parity in the gravitational action as an explanation of some astrophysical observations [10]. Therefore it may be interesting to have a consistent description of the models which are capable to explain such a violation, and the study of renormalization of the model with axial scalar may be a useful step in better understanding of a possible quantum origin of such terms.

Another interesting aspect of the model under consideration is that such a theory has two different scalar masses, that is a usual situation in effective field theory (see e.g. the book [11]). In the recent work [12] we explored the non-local finite contributions of the curved-spacetime diagrams with mixed internal lines, e.g. one of a light and another of a heavy scalar fields. Here we supplement this result by deriving the effective potential in the two-scalar model. It is worth pointing out that this situation is typical for effective field theories, especially the ones with different mass scales and diagrams with mixed types of internal lines. The effective potential involves two independent contributions, one from the loops of scalar fields and another one from the spinor loop. In what follows we show that the results for these contributions look somehow unusual. In the scalar sector we meet a complicated non-polynomial mixing of the scalar masses and couplings, something one could expect for the two-scalar model.

The paper is organized as follows. In Sect. 2 we describe the model including a real scalar field and a pseudoscalar field coupled to $N$-component fermionic field and derive the corresponding one-loop divergences. The one-loop renormalization relations in this theory and the derivation of the renormalization group functions are collected in Sect. 3. In Sect. 4 the renormalized one-loop effective potential is derived by using the local momentum representation. Finally, our conclusions and the discussion of the results are presented in Sect. 5.

\section{Yukawa model and its renormalization}

Consider a Yukawa model including a real scalar field $\varphi$ and a real pseudoscalar (axial scalar) field $\chi$, coupled to the $N$ copies of a fermionic field $\Psi_{i}$, with the classical action of the form

$$
\begin{aligned}
S= & \int d^{4} x \sqrt{-g}\left\{\bar{\Psi}_{i}\left(i \not \nabla-M-h_{1} \varphi-h_{2} \chi \gamma^{5}\right) \delta^{i j} \Psi_{j}\right. \\
& +\frac{1}{2} g^{\mu \nu} \partial_{\mu} \varphi \partial_{\nu} \varphi+\frac{1}{2} g^{\mu \nu} \partial_{\mu} \chi \partial_{\nu} \chi-\frac{1}{2} m_{1}^{2} \varphi^{2}-\frac{1}{2} m_{2}^{2} \chi^{2} \\
& +\frac{1}{2} \xi_{1} R \varphi^{2}+\frac{1}{2} \xi_{2} R \chi^{2}-\frac{\lambda_{1}}{4 !} \varphi^{4}-\frac{\lambda_{2}}{4 !} \chi^{4} \\
& \left.-\frac{\lambda_{3}}{2} \varphi^{2} \chi^{2}-\frac{g}{3 !} \varphi^{3}-\frac{p}{2} \varphi \chi^{2}-\tau \varphi-f R \varphi\right\},
\end{aligned}
$$

where $m_{1}, m_{2}$ and $M$ are respectively the masses of scalar, pseudoscalar and spinor fields, $h_{1}$ and $h_{2}$ are the Yukawa coupling constants. Finally, $\lambda_{1}, \lambda_{2}, \lambda_{3}, g, p$ and $\tau$ are coupling constants in the scalar - pseudoscalar sectors, that survive in the flat limit, while $\xi_{1}$ and $f$ are the nonminimal parameters of the scalar field and $\xi_{2}$ the nonminimal parameter of the interaction between axial scalar field with gravity. It is easy to note that the action has not only the standard even terms, but also a set of odd terms, with the dimensional parameters $g, p$ and $f$. As we shall see in brief, these terms are necessary to achieve renormalizability of the theory. The last observation is that term which are linear and cubic in the pseudoscalar field are excluded by the requirement that the Lagrangian is a parity-even scalar.

In order to calculate the one-loop divergences, we shall use the heat-kernel method, and perform the background quantum splitting of the fields, according to

$$
\begin{aligned}
& \varphi \rightarrow \varphi+\sigma, \quad \chi \rightarrow \chi+\rho, \\
& \bar{\Psi}_{i} \rightarrow \bar{\Psi}_{i}+\bar{\eta}_{i}, \quad \Psi_{j} \rightarrow \Psi_{j}+\eta_{j},
\end{aligned}
$$

where $\varphi, \chi, \bar{\Psi}, \Psi$ are the classical background fields and $\sigma, \rho, \bar{\eta}, \eta$ their quantum counterparts.

The bilinear in quantum fields part of the action is written as follows

$$
\begin{aligned}
S^{(2)}= & \frac{1}{2} \int d^{4} x \sqrt{-g}\left(\begin{array}{lll}
\sigma & \rho & \bar{\eta}_{i}
\end{array}\right) \hat{H}\left(\begin{array}{c}
\sigma \\
\rho \\
\eta_{j}
\end{array}\right) \\
= & \frac{1}{2} \int d^{4} x \sqrt{-g}\left\{\sigma H_{11} \sigma+\rho H_{21} \sigma+\bar{\eta}_{i} H_{31} \sigma\right. \\
& +\sigma H_{12} \rho+\rho H_{22} \rho+\bar{\eta}_{i} H_{32} \rho+\sigma H_{13} \eta_{j} \\
& \left.+\rho H_{23} \eta_{j}+\bar{\eta}_{i} H_{33} \eta_{j}\right\},
\end{aligned}
$$

where the elements of the matrix operator $\hat{H}$ have the form

$$
\begin{aligned}
& H_{11}=-\square-m_{1}^{2}+\xi_{1} R-g \varphi-\lambda_{3} \chi^{2}-\frac{\lambda_{1}}{2} \varphi^{2}, \\
& H_{12}=-4 \varphi \chi \lambda_{3}-2 p \chi, \\
& H_{13}=-2 h_{1} \bar{\Psi}_{j}, \\
& H_{21}=-4 \varphi \chi \lambda_{3}-2 p \chi, \\
& H_{22}=-\square-m_{2}^{2}+\xi_{2} R-p \varphi-\lambda_{3} \varphi^{2}-\frac{\lambda_{2}}{2} \chi^{2}, \\
& H_{23}=-2 h_{2} \bar{\Psi}_{j} \gamma^{5}, \\
& H_{31}=-2 h_{1} \Psi_{i}, \\
& H_{32}=-2 h_{2} \gamma^{5} \Psi_{i}, \\
& H_{33}=2\left(i \not \nabla-M-h_{1} \varphi-h_{2} \chi \gamma^{5}\right) \delta^{i j} .
\end{aligned}
$$

It proves useful to introduce conjugated matrix operator

$$
\hat{H}^{*}=\left(\begin{array}{ccc}
-1 & 0 & 0 \\
0 & -1 & 0 \\
0 & 0 & -\frac{1}{2}(i \not \nabla+M)
\end{array}\right) \text {. }
$$


The one-loop quantum contribution to effective action is defined by the expression $\operatorname{Tr} \ln (\hat{H})$. To calculate the divergences of effective action we will write it as

$\operatorname{Tr} \ln (\hat{H})=\operatorname{Tr} \ln \left(\hat{H} \hat{H}^{*}\right)-\operatorname{Tr} \ln \left(\hat{H}^{*}\right)$.

The last term $\operatorname{Tr} \ln \hat{H}^{*}$ contributes only to the vacuum divergences that are known for an arbitrary model $[6,13]$. Therefore it is sufficient to calculate the divergences of the product $\hat{H} \hat{H}^{*}$, that has a standard form,

$\hat{\mathcal{H}}=\hat{H} \hat{H}^{*}=\hat{1} \square+2 \hat{h}^{\mu} \nabla_{\mu}+\hat{\Pi}$.

Hence,

$$
\begin{aligned}
\mathcal{H}_{11}= & \square+m_{1}^{2}+\frac{\lambda_{1}}{2} \varphi^{2}-\xi_{1} R+\lambda_{3} \chi^{2}+g \varphi, \\
\mathcal{H}_{12}= & 2 p \chi+4 \lambda_{3} \varphi \chi, \\
\mathcal{H}_{13}= & h_{1} \bar{\Psi}_{j}(i \not \nabla+M), \\
\mathcal{H}_{21}= & 2 p \chi+4 \lambda_{3} \varphi \chi, \\
\mathcal{H}_{22}= & \square+m_{2}^{2}+\frac{\lambda_{2}}{2} \chi^{2}-\xi_{2} R+\lambda_{3} \varphi^{2}+p \varphi, \\
\mathcal{H}_{23}= & h_{2} \bar{\Psi}_{j} \gamma^{5}(i \not \nabla+M), \\
\mathcal{H}_{31}= & 2 h_{1} \Psi_{i}, \\
\mathcal{H}_{32}= & 2 h_{2} \gamma^{5} \Psi_{i}, \\
\mathcal{H}_{33}= & \delta^{i j}\left[\square-\frac{1}{4} R+M^{2}+h_{1} \varphi(i \not \nabla+M)\right. \\
& \left.+h_{2} \chi \gamma^{5}(i \not \nabla+M)\right] .
\end{aligned}
$$

where we can identify

$$
\begin{aligned}
& h_{13}^{\mu}=\frac{i h_{1}}{2} \bar{\Psi}_{j} \gamma^{\mu}, \quad h_{23}^{\mu}=\frac{i h_{2}}{2} \bar{\Psi}_{j} \gamma^{5} \gamma^{\mu}, \\
& h_{33}^{\mu}=\frac{i}{2}\left(h_{1} \varphi+h_{2} \chi \gamma^{5}\right) \gamma^{\mu} \delta^{i j}
\end{aligned}
$$

and

$$
\begin{aligned}
& \Pi_{11}=m_{1}^{2}+\frac{\lambda_{1}}{2} \varphi^{2}-\xi_{1} R+g \varphi+\lambda_{3} \chi^{2}, \\
& \Pi_{12}=2 p \chi+4 \lambda_{3} \varphi \chi \\
& \Pi_{13}=h_{1} M \bar{\Psi}_{j} \\
& \Pi_{21}=2 p \chi+4 \lambda_{3} \varphi \chi \\
& \Pi_{22}=m_{2}^{2}+\frac{\lambda_{2}}{2} \chi^{2}-\xi_{2} R+p \varphi+\lambda_{3} \varphi^{2}, \\
& \Pi_{23}=h_{2} M \bar{\Psi}_{j} \gamma^{5} \\
& \Pi_{31}=2 h_{1} \Psi_{i}, \\
& \Pi_{32}=2 h_{2} \gamma^{5} \Psi_{i}, \\
& \Pi_{33}=\delta^{i j}\left[M^{2}-\frac{1}{4} R+h_{1} M \varphi+h_{2} M \chi \gamma^{5}\right] .
\end{aligned}
$$

The Schwinger-De-Witt proper-time (heat kernel) technique [14] yields the general expression for the one-loop divergences in the form

$$
\begin{aligned}
\Gamma_{\text {div }}^{(1)}= & -\frac{\mu^{D-4}}{\varepsilon} \int d^{D} x \sqrt{-g} \operatorname{sir}\left\{\frac{1}{2} \hat{P}^{2}+\frac{1}{12} \hat{S}_{\mu \nu}^{2}\right. \\
& \left.+\frac{1}{6} \square \hat{P}+\frac{\hat{1}}{180}\left(R_{\mu \nu \alpha \beta}^{2}-R_{\mu \nu}^{2}+\square R\right)\right\},
\end{aligned}
$$

where $\varepsilon=(4 \pi)^{2}(D-4)$ and

$$
\begin{aligned}
\hat{P} & =\hat{\Pi}+\frac{\hat{1}}{6} R-\nabla_{\mu} \hat{h}^{\mu}-\hat{h}_{\mu} \hat{h}^{\mu}, \\
\hat{S}_{\mu \nu} & =\left[\nabla_{\nu}, \nabla_{\mu}\right] \hat{1}+\nabla_{\nu} \hat{h}_{\mu}-\nabla_{\mu} \hat{h}_{\nu}+\hat{h}_{\nu} \hat{h}_{\mu}-\hat{h}_{\mu} \hat{h}_{\nu} .
\end{aligned}
$$

The relations (11), (12) lead to the following result for the one-loop divergences in the model under consideration, ${ }^{1}$

$\Gamma_{d i v}^{(1)}=\Gamma_{v a c, d i v}^{(1)}+\Gamma_{m, d i v}^{(1)}$,

where

$$
\begin{aligned}
\Gamma_{\text {vac,div }}^{(1)}= & -\frac{\mu^{D-4}}{\varepsilon} \int d^{D} x \sqrt{-g}\left\{\frac{1}{2}\left(m_{1}^{4}+m_{2}^{4}\right)\right. \\
& -2 N M^{4}+\left(\frac{N}{3} M^{2}-m_{1}^{2} \tilde{\xi}_{1}-m_{2}^{2} \tilde{\xi}_{2}\right) R \\
& +\frac{1}{2}\left(\tilde{\xi}_{1}^{2}+\tilde{\xi}_{2}^{2}-\frac{N}{9}\right) R^{2}+\frac{8 N-2}{180} R_{\mu \nu}^{2} \\
& \left.+\left(\frac{N}{24}+\frac{1}{45}\right) R_{\mu \nu \alpha \beta}^{2}+\left(\frac{1}{45}+\frac{N}{9}-\frac{\tilde{\xi}_{1}+\tilde{\xi}_{2}}{6}\right) \square R\right\}
\end{aligned}
$$

and

$$
\begin{aligned}
\Gamma_{m, d i v}^{(1)}= & -\frac{\mu^{D-4}}{\varepsilon} \int d^{D} x \sqrt{-g}\left\{\sum _ { k } 3 \overline { \Psi } _ { k } \left[\frac{i}{2} h_{1}^{2} \not \nabla\right.\right. \\
& -\frac{i}{2} h_{2}^{2} \not \nabla+h_{1}^{2}\left(M+h_{1} \varphi-h_{2} \chi \gamma^{5}\right) \\
& \left.+h_{2}^{2}\left(M+h_{1} \varphi-h_{2} \chi \gamma^{5}\right)\right] \Psi_{k}+2 N h_{1}^{2}\left(\partial_{\mu} \varphi\right)^{2} \\
& -2 N h_{2}^{2}\left(\partial_{\mu} \chi\right)^{2}+\left(\frac{1}{3} N h_{1}^{2}-\frac{\lambda_{1}}{2} \tilde{\xi}_{1}-\lambda_{3} \tilde{\xi}_{2}\right) R \varphi^{2} \\
& +\left(\frac{1}{8} \lambda_{1}^{2}+\frac{1}{2} \lambda_{3}^{2}-2 N h_{1}^{4}\right) \varphi^{4}+\left[\frac{2}{3} N h_{1} M-g \tilde{\xi}_{1}\right. \\
& \left.-p \tilde{\xi}_{2}\right] R \varphi+\left(m_{1}^{2} g+m_{2}^{2} p-8 N h_{1} M^{3}\right) \varphi \\
& +\frac{1}{2}\left(g^{2}+p^{2}+\lambda_{1} m_{1}^{2}+2 \lambda_{3} m_{2}^{2}-24 N h_{1}^{2} M^{2}\right) \varphi^{2} \\
& +\left(\lambda_{3} p-8 N M h_{1}^{3}+\frac{1}{2} g \lambda_{1}\right) \varphi^{3} \\
& +\frac{1}{2}\left(2 \lambda_{3} m_{1}^{2}+\lambda_{2} m_{2}^{2}+8 p^{2}+8 N h_{2}^{2} M^{2}\right) \chi^{2} \\
& +\left(\frac{1}{2} \lambda_{3}^{2}+\frac{1}{8} \lambda_{2}^{2}-2 N h_{2}^{4}\right) \chi^{4} \\
& -\frac{1}{2}\left(\lambda_{2} \tilde{\xi}_{2}+2 \lambda_{3} \tilde{\xi}_{1}+\frac{2 N}{3} h_{2}^{2}\right) R \chi^{2}
\end{aligned}
$$

$\overline{1}$ We present here only the final result, the intermediate formulas can be found in the "Appendix". 


$$
\begin{aligned}
& +\frac{1}{2}\left(\lambda_{1} \lambda_{3}+\lambda_{2} \lambda_{3}+8 N h_{1}^{2} h_{2}^{2}+32 \lambda_{3}^{2}\right) \varphi^{2} \chi^{2} \\
& +\left(g \lambda_{3}+\frac{1}{2} p \lambda_{2}+16 p \lambda_{3}+8 N h_{1} h_{2}^{2} M\right) \varphi \chi^{2} \\
& +\frac{1}{6}\left(g+p-8 N h_{1} M\right) \square \varphi \\
& +\frac{1}{12}\left(\lambda_{1}+2 \lambda_{3}-16 N h_{1}^{2}\right) \square \varphi^{2} \\
& \left.+\frac{1}{12}\left(\lambda_{2}+2 \lambda_{3}+16 N h_{2}^{2}\right) \square \chi^{2}\right\}
\end{aligned}
$$

For compactness, we have introduced the notations $\tilde{\xi}_{1,2}=$ $\xi_{1,2}-\frac{1}{6}$. The vacuum divergences are included for the sake of completeness.

The expression (15) shows that the odd terms, which we have included in the classical action (1), subject to the divergences. Exactly as it is the case in the simpler single-scalar theory, these terms have no symmetry protection and the structure of divergences is exactly as should be expected from the symmetry and power-counting arguments.

\section{Renormalization}

Once the form of the one-loop divergences is known one can easily find the relations between bare and renormalizable quantities. For the fields we meet

$$
\begin{aligned}
\varphi_{0} & =\mu^{\frac{D-4}{2}}\left(1+\frac{2 N h_{1}^{2}}{\epsilon}\right) \varphi, \\
\chi_{0} & =\mu^{\frac{D-4}{2}}\left(1-\frac{2 N h_{2}^{2}}{\epsilon}\right) \chi, \\
\Psi_{k 0} & =\mu^{\frac{D-4}{2}}\left[1+\frac{3}{4 \epsilon}\left(h_{1}^{2}-h_{2}^{2}\right)\right] \Psi_{k} .
\end{aligned}
$$

The relations for masses have the form

$$
\begin{aligned}
M_{0}= & \left(1-\frac{9}{2 \epsilon} h_{1}^{2}-\frac{3}{2 \epsilon} h_{2}^{2}\right) M, \\
m_{10}^{2}= & m_{1}^{2}-\frac{1}{\epsilon}\left(g^{2}+p^{2}+4 N h_{1}^{2} m_{1}^{2}+\lambda_{1} m_{1}^{2}+2 \lambda_{3} m_{2}^{2}\right. \\
& \left.-24 N h_{1}^{2} M^{2}\right), \\
m_{20}^{2}= & m_{2}^{2}-\frac{1}{\epsilon}\left(8 p^{2}-4 N h_{2}^{2} m_{2}^{2}+\lambda_{2} m_{2}^{2}+2 \lambda_{3} m_{1}^{2}\right. \\
& \left.+8 N h_{2}^{2} M^{2}\right) .
\end{aligned}
$$

For the even couplings and nonminimal parameters we find

$$
\begin{aligned}
& \xi_{10}=\xi_{1}-\frac{\lambda_{1}+4 N h_{1}^{2}}{\epsilon} \tilde{\xi}_{1}-\frac{2 \lambda_{3}}{\epsilon} \tilde{\xi}_{2}, \\
& \xi_{20}=\xi_{2}+\frac{4 N h_{2}^{2}-\lambda_{2}}{\epsilon} \tilde{\xi}_{2}-\frac{2}{\epsilon} \lambda_{3} \tilde{\xi}_{1}, \\
& h_{10}=\mu^{\frac{4-D}{2}} h_{1}\left(1-\frac{4 N h_{1}^{2}+9 h_{1}^{2}+3 h_{2}^{2}}{2 \epsilon}\right),
\end{aligned}
$$

$$
\begin{aligned}
h_{20}= & \mu^{\frac{4-D}{2}} h_{2}\left(1+\frac{4 N h_{2}^{2}+9 h_{2}^{2}+3 h_{1}^{2}}{2 \epsilon}\right), \\
\lambda_{10}= & \mu^{4-D}\left(\lambda_{1}+\frac{48 N h_{1}^{4}-8 N \lambda_{1} h_{1}^{2}-3 \lambda_{1}^{2}-12 \lambda_{3}^{2}}{\epsilon}\right), \\
\lambda_{20}= & \mu^{4-D}\left(\lambda_{2}+\frac{48 N h_{2}^{4}+8 N \lambda_{2} h_{2}^{2}-3 \lambda_{2}^{2}-12 \lambda_{3}^{2}}{\epsilon}\right), \\
\lambda_{30}= & \mu^{4-D}\left(\lambda_{3}-\frac{1}{\epsilon}\left(\lambda_{1} \lambda_{3}+\lambda_{2} \lambda_{3}+8 N h_{1}^{2} h_{2}^{2}+32 \lambda_{3}^{2}\right.\right. \\
& \left.\left.+4 N \lambda_{3} h_{1}^{2}-4 N \lambda_{3} h_{2}^{2}\right)\right) .
\end{aligned}
$$

And, finally, for the odd couplings and nonminimal parameters,

$$
\begin{aligned}
g_{0}= & \mu^{\frac{4-D}{2}}\left(g+\frac{48 N M h_{1}^{3}-3 g \lambda_{1}-6 N h_{1}^{2} g-6 \lambda_{3} p}{\epsilon}\right), \\
\tau_{0}= & \mu^{\frac{4-D}{2}}\left(\tau+\frac{8 N h_{1} M^{3}-2 N \tau h_{1}^{2}-m_{1}^{2} g-m_{2}^{2} p}{\epsilon}\right), \\
p_{0}= & \mu^{\frac{D-4}{2}}\left(p-\frac{1}{\epsilon}\left(2 \lambda_{3} g+\lambda_{2} p+32 \lambda_{3} p+16 N h_{1} h_{2}^{2} M\right.\right. \\
& \left.\left.-4 N h_{2}^{2} p+2 N h_{1}^{2} p\right)\right), \\
f_{0}= & \mu^{\frac{D-4}{2}}\left[f+\frac{g}{\epsilon} \tilde{\xi}_{1}+\frac{p}{\epsilon} \tilde{\xi}_{2}-\frac{2 N h_{1} M+6 N f h_{1}^{2}}{3 \epsilon}\right] .
\end{aligned}
$$

Note the non-trivial renormalization of the odd coupling parameters and in particular of the new non-minimal coupling parameter $f$.

The $\beta$ - and $\gamma$-functions can be calculated from the renormalization relations for the parameters and fields. For the theories in curved spacetime the procedure $[15,16]$ is described in detail in the book [6], so we give only the final results for

$$
\begin{aligned}
\beta_{P} & =\lim _{D \rightarrow 4} \mu \frac{d P}{d \mu}, \\
\gamma_{\Phi} \Phi & =\lim _{D \rightarrow 4} \mu \frac{d \Phi}{d \mu},
\end{aligned}
$$

where $P=\left(m_{1}^{2}, m_{2}^{2}, M, h_{1}, h_{2}, \lambda_{1}, \lambda_{2}, \xi_{1}, \xi_{2}, g, p, \tau, f\right)$ are the renormalized parameters and $\Phi=\left(\varphi, \chi, \Psi_{k}\right)$ are the renormalized fields. Using the relations (16)-(19), we obtain the following results:

$$
\begin{aligned}
& \beta_{h_{1}}=\frac{\left(4 N h_{1}^{3}+9 h_{1}^{3}+3 h_{1} h_{2}^{2}\right)}{2(4 \pi)^{2}}, \\
& \beta_{h_{2}}=-\frac{\left(4 N h_{2}^{3}+9 h_{2}^{3}+3 h_{1}^{2} h_{2}\right)}{2(4 \pi)^{2}}, \\
& \beta_{M}=\frac{3 M}{2(4 \pi)^{2}}\left(3 h_{1}^{2}+h_{2}^{2}\right), \\
& \beta_{\lambda_{1}}=\frac{1}{(4 \pi)^{2}}\left(3 \lambda_{1}^{2}+8 N \lambda_{1} h_{1}^{2}-48 N h_{1}^{4}+12 \lambda_{3}^{2}\right),
\end{aligned}
$$




$$
\begin{aligned}
\beta_{\lambda_{2}}= & \frac{1}{(4 \pi)^{2}}\left(3 \lambda_{2}^{2}-8 N \lambda_{2} h_{2}^{2}-48 N h_{2}^{4}+12 \lambda_{3}^{2}\right), \\
\beta_{\lambda_{3}}= & \frac{1}{(4 \pi)^{2}}\left(\lambda_{1} \lambda_{3}+\lambda_{2} \lambda_{3}+32 \lambda_{3}^{2}+8 N h_{1}^{2} h_{2}^{2}\right. \\
& \left.+4 N \lambda_{3} h_{1}^{2}-4 N \lambda_{3} h_{2}^{2}\right), \\
\beta_{\xi_{1}}= & \frac{1}{(4 \pi)^{2}}\left[\left(\lambda_{1}+4 N h_{1}^{2}\right)\left(\xi_{1}-\frac{1}{6}\right)+2 \lambda_{3}\left(\xi_{2}-\frac{1}{6}\right)\right], \\
\beta_{\xi_{2}}= & \frac{1}{(4 \pi)^{2}}\left[\left(\lambda_{2}-4 N h_{2}^{2}\right)\left(\xi_{2}-\frac{1}{6}\right)+2 \lambda_{3}\left(\xi_{1}-\frac{1}{6}\right)\right], \\
\beta_{g}= & \frac{1}{(4 \pi)^{2}}\left(3 g \lambda_{1}+6 N g h_{1}^{2}-48 N M_{1}^{3}+6 p \lambda_{3}\right), \\
\beta_{p}= & \frac{1}{(4 \pi)^{2}}\left(p \lambda_{2}+32 \lambda_{3} p-4 N p h_{2}^{2}+2 N p h_{1}^{2}\right. \\
& \left.+2 g \lambda_{3}+16 N M h_{1} h_{2}^{2}\right), \\
\beta_{m_{1}^{2}}= & \frac{1}{(4 \pi)^{2}}\left[m_{1}^{2} \lambda_{1}+g^{2}+p^{2}+\left(4 m_{1}^{2}-24 M^{2}\right) N h_{1}^{2}\right. \\
& \left.+2 \lambda_{3} m_{2}^{2}\right], \\
\beta_{\tau}= & \frac{1}{(4 \pi)^{2}}\left(2 N \tau h_{1}^{2}+g m_{1}^{2}+p m_{2}^{2}-8 N h_{1} M^{3}\right), \\
\beta_{m_{2}^{2}=} & \frac{1}{(4 \pi)^{2}}\left[m_{2}^{2} \lambda_{2}+8 p^{2}+\left(8 M^{2}-4 m_{2}^{2}\right) N h_{2}^{2}\right. \\
& \left.+2 \lambda_{3} m_{1}^{2}\right], \\
& \left.+\frac{2}{3} N h_{1}\right] . \\
& \\
&
\end{aligned}
$$

For the $\gamma$-functions we have

$$
\begin{aligned}
\gamma_{\varphi} & =-\frac{2 N h_{1}^{2}}{(4 \pi)^{2}}, \quad \gamma_{\chi}=\frac{2 N h_{2}^{2}}{(4 \pi)^{2}}, \\
\gamma_{\Psi_{k}} & =\frac{3}{4(4 \pi)^{2}}\left(h_{2}^{2}-h_{1}^{2}\right) .
\end{aligned}
$$

A good check is that, if considering the conformal invariant theory, with vanishing masses and other dimensional constants, $g, p, \tau$ and $f$, and assuming $\xi_{1}=\xi_{2}=\frac{1}{6}$, the pole coefficient in the expression for the divergences (15) is also conformal invariant. Consequently, the $\beta$-functions for $\xi_{1}$ and $\xi_{2}$ in this case are linear combinations of $\tilde{\xi}_{1}$ and $\tilde{\xi}_{2}$, defined after Eq. (14).

\section{Effective potential}

In this section we derive the one-loop effective potential in the model under consideration up to first order in scalar curvature, using the local momentum representation, based on the Riemann normal coordinates. This method is quite efficient for mass-dependent calculations of local quantities, such as the effective potential.

The effective potential $V_{\text {eff }}(\varphi)$ is defined as the zerothorder term in the derivative expansion of the effective action of a background scalar field $\varphi(x)$,

$$
\begin{aligned}
\Gamma\left[\varphi, g_{\mu \nu}\right]= & \int d^{D} x \sqrt{-g}\left\{-V_{e f f}(\varphi)\right. \\
& \left.+\frac{1}{2} Z(\varphi) g^{\mu \nu} \partial_{\mu} \varphi \partial_{\nu} \varphi+\cdots\right\},
\end{aligned}
$$

where $D$ is the spacetime dimension.

Within the loop expansion of the effective action, the corresponding one-loop correction to the effective potential is given by

$\int d^{D} x \sqrt{-g} V(\varphi)=\left.\frac{1}{2} \sin \ln \hat{H}\right|_{\varphi=\text { const }}$

where $\hat{H}$ is the bilinear operator of action (3).

The curvature expansion of $V(\varphi)$ reads

$V=V_{0}+V_{1}+\cdots$

where $V_{0}^{(1)}$ is the well-known flat-spacetime effective potential, which has been derived many times and in different ways starting from the work of Coleman and Weinberg [7] and $V_{1}$ is the first order in scalar curvature $R$. In curved spacetime the potential can also be derived in different ways.

Let us emphasize that in all known examples the effective potential can be obtained by solving the renormalization group equation for the effective action, in both flat [7] and curved [8] spacetimes (see e.g. Ref. [6] for detailed introduction and further references. The generalization to the model with a single sterile scalar proceeds is done in a close analogy to the standard approach, but with some modification due to the presence of the odd interaction terms [4].

The renormalization group equation for the the effective action has the form $[6,15]$

$$
\begin{aligned}
& \left\{\mu \frac{\partial}{\partial \mu}+\beta_{P} \frac{\partial}{\partial P}+\int d^{D} x \gamma_{\Phi} \Phi \frac{\delta}{\delta \Phi(x)}\right\} \\
& \quad \times \Gamma\left[g_{\alpha \beta}, \Phi, P, D, \mu\right]=0
\end{aligned}
$$

where we assume the sum over all parameters (couplings and masses) $P$ and the fields $\Phi=\left(\varphi, \chi, \Psi_{k}\right)$. The effective potential satisfies the same equation, due to the separation of different terms in (24). Then, the result for, e.g., a single scalar field can be presented as a general symbolic expression ${ }^{2}$

\footnotetext{
${ }^{2}$ We will not write down similar formula for the theory (1), because it is too long. The interested reader can easily obtain it by analogy with Eq. (28).
} 


$$
\begin{aligned}
V_{e f f}= & -\frac{1}{2} m^{2} \varphi^{2}-\frac{1}{2} \xi R \varphi^{2}+\frac{\lambda}{4 !} \varphi^{4}+\frac{g}{3 !} \varphi^{3}+\tau \varphi+f R \varphi \\
& -\frac{1}{4} \varphi^{2}\left(\beta_{m^{2}}+2 m^{2} \gamma_{\varphi}\right)\left[\ln \left(\frac{\varphi_{1 *}^{2}}{\mu^{2}}\right)+C_{1}\right] \\
& -\frac{1}{4} R \varphi^{2}\left(\beta_{\xi}+2 \xi \gamma_{\varphi}\right)\left[\ln \left(\frac{\varphi_{2 *}^{2}}{\mu^{2}}\right)+C_{2}\right] \\
& +\frac{1}{12} \varphi^{3}\left(\beta_{g}+3 g \gamma_{\varphi}\right)\left[\ln \left(\frac{\varphi_{3 *}^{2}}{\mu^{2}}\right)+C_{3}\right] \\
& +\frac{1}{48} \varphi^{4}\left(\beta_{\lambda}+4 \lambda \gamma_{\varphi}\right)\left[\ln \left(\frac{\varphi_{4 *}^{2}}{\mu^{2}}\right)+C_{4}\right] \\
& +\frac{1}{2} \varphi\left(\beta_{\tau}+\tau \gamma_{\varphi}\right)\left[\ln \left(\frac{\varphi_{5 *}^{2}}{\mu^{2}}\right)+C_{5}\right] \\
& +\frac{1}{2} R \varphi\left(\beta_{f}+f \gamma_{\varphi}\right)\left[\ln \left(\frac{\varphi_{6 *}^{2}}{\mu^{2}}\right)+C_{6}\right],
\end{aligned}
$$

where all beta- and gamma-functions are given in Eqs. (22) and (23). The set of the constants $C_{1 \ldots 6}$ in the last expression (28) can be found from the initial renormalization conditions. For instance, the two well-known values, corresponding to the standard choices in the massless scalar case are $C_{4}=-\frac{25}{6}$ obtained in [7] and $C_{2}=-3$ obtained in [6,8].

The symbolic expressions $\ln \left(\frac{\varphi_{k *}^{2}}{\mu^{2}}\right)$ with $k=1,2, \ldots, 6$, in the formula (28) depend on the theory under consideration. For instance, in the model with a single sterile scalar [4], these quantities appear as linear combinations of the logarithms

$t^{(0)}=\frac{1}{2} \ln \left[\frac{m^{2}+\frac{1}{2} \lambda \varphi^{2}+g \varphi}{\mu^{2}}\right]$

and

$t^{\left(\frac{1}{2}\right)}=\frac{1}{2} \ln \left[\frac{(M+h \varphi)^{2}}{\mu^{2}}\right]$

for the scalar and fermion contributions to the effective potential, correspondingly. Namely, the logarithms (29) and (30) are used as an efficient Ansatz to solve the renormalization group equation for the effective potential.

In the massless case or in the limit of large-scalar limit, one should expect that the asymptotic behavior of all terms should be

$\ln \left(\frac{\varphi_{k *}^{2}}{\mu^{2}}\right) \propto \ln \left(\frac{\varphi^{2}}{\mu^{2}}\right)$.

In the subsequent subsection we perform direct calculation of the scalar contribution in the model (1) and show that the result is inconsistent with the expectation based on the Ansatz that consists in guessing the form of the logarithms, such as (29) and (30).

The calculations presented below were performed in the covariant cut-off regularization of the Euclidean integrals in the local momentum representation. In the case of effective potential this regularization is the simplest options. On the other hand, the transition to the covariant cut-off in the proper time integral, and consequently to the dimensional regularization is automatic, as discussed in [18] (see also earlier general investigation in flat spacetime [19]).

\subsection{Two-scalar sector}

Let us start from the bilinear form of the action in the scalar sector of (3) in the form

$S_{0}^{(2)}=\frac{1}{2} \int d^{4} x \sqrt{-g}\left(\begin{array}{ll}\sigma & \rho\end{array}\right) \hat{H}_{s}\left(\begin{array}{c}\sigma \\ \rho\end{array}\right)$,

where the matrix operator has the form

$\hat{H}_{s}=\square \hat{\imath}+\left(\begin{array}{ll}M_{11}^{2} & M_{12}^{2} \\ M_{21}^{2} & M_{22}^{2}\end{array}\right)$

with $\hat{1}=\operatorname{diag}(1,1)$ and

$M_{11}^{2}=\tilde{m}_{1}^{2}-\xi_{1} R$,

$M_{22}^{2}=\tilde{m}_{2}^{2}-\xi_{2} R$,

$M_{12}^{2}=M_{21}^{2}=2 p \chi+4 \lambda_{3} \varphi \chi$,

where

$\tilde{m}_{1}^{2}=m_{1}^{2}+\frac{\lambda_{1}}{2} \varphi^{2}+\lambda_{3} \chi^{2}+g \varphi$,

$\tilde{m}_{2}^{2}=m_{2}^{2}+\frac{\lambda_{2}}{2} \chi^{2}+\lambda_{3} \varphi^{2}+p \varphi$.

In order to simplify the calculations let us diagonalize the matrix in the second term of relation (33), by making a rotation in the space of the fields,

$\left(\begin{array}{c}\sigma \\ \rho\end{array}\right)=U\left(\begin{array}{l}\phi_{1} \\ \phi_{2}\end{array}\right)$, where $U=\left(\begin{array}{cc}\cos \alpha & -\sin \alpha \\ \sin \alpha & \cos \alpha\end{array}\right)$.

After this transformation, Eq. (32) becomes

$$
\begin{aligned}
S_{0}^{(2)}= & \frac{1}{2} \int d^{4} x \sqrt{-g}\left\{\phi_{1} \square \phi_{1}+\phi_{2} \square \phi_{2}\right. \\
& +\phi_{1}\left[\cos ^{2}(\alpha) M_{11}^{2}+\sin ^{2}(\alpha) M_{22}^{2}-\sin (2 \alpha) M_{12}^{2}\right] \phi_{1} \\
& +\phi_{2}\left[\sin ^{2}(\alpha) M_{11}^{2}+\cos ^{2}(\alpha) M_{22}^{2}+\sin (2 \alpha) M_{12}^{2}\right] \phi_{2} \\
& \left.+\phi_{1}\left[\sin (2 \alpha)\left(M_{22}^{2}-M_{11}^{2}\right)+2 \cos (2 \alpha) M_{12}^{2}\right] \phi_{2}\right\} .
\end{aligned}
$$

Now, we can simply choose

$$
\begin{aligned}
& \cos (2 \alpha)=\frac{M_{22}^{2}-M_{11}^{2}}{2 M_{12}^{2}} \sin (2 \alpha) \Longrightarrow \\
& \cot (2 \alpha)=\Theta=\frac{M_{22}^{2}-M_{11}^{2}}{2 M_{12}^{2}},
\end{aligned}
$$


such that the last term in (38) vanishes and the new diagonal matrix $\hat{\mathcal{H}}_{s}=U^{-1} \hat{H}_{S} U$ has the form

$$
\begin{aligned}
\hat{\mathcal{H}}_{s} & =\square \hat{1} \\
& +\left(\begin{array}{cc}
a M_{11}^{2}+b M_{22}^{2}-c M_{12}^{2} & 0 \\
0 & b M_{11}^{2}+a M_{22}^{2}+c M_{12}^{2}
\end{array}\right),
\end{aligned}
$$

where

$$
\begin{aligned}
& a=\frac{1}{2}+\frac{\Theta}{2 \sqrt{1+\Theta^{2}}}, \quad b=\frac{1}{2}-\frac{\Theta}{2 \sqrt{1+\Theta^{2}}}, \\
& c=\frac{1}{\sqrt{1+\Theta^{2}}} .
\end{aligned}
$$

Since we are interested in the $\mathcal{O}(R)$-approximation, it is useful to rewrite (40) as

$\hat{\mathcal{H}}_{s}=\left(\begin{array}{cc}\square-\Pi_{1}-\zeta_{1} R+\cdots & 0 \\ 0 & \square-\Pi_{2}-\zeta_{2} R+\cdots\end{array}\right)$,

where $\cdots$ stands for higher orders in $R$ with

$$
\begin{aligned}
\Pi_{1}= & -\left(\frac{1}{2}+\frac{\Theta_{0}}{2 \sqrt{1+\Theta_{0}^{2}}}\right) \tilde{m}_{1}^{2} \\
& -\left(\frac{1}{2}-\frac{\Theta_{0}}{2 \sqrt{1+\Theta_{0}^{2}}}\right) \tilde{m}_{2}^{2}+\frac{M_{12}^{2}}{\sqrt{1+\Theta_{0}^{2}}}, \\
\Pi_{2}= & -\left(\frac{1}{2}-\frac{\Theta_{0}}{2 \sqrt{1+\Theta_{0}^{2}}}\right) \tilde{m}_{1}^{2} \\
& -\left(\frac{1}{2}+\frac{\Theta_{0}}{2 \sqrt{1+\Theta_{0}^{2}}}\right) \tilde{m}_{2}^{2}-\frac{M_{12}^{2}}{\sqrt{1+\Theta_{0}^{2}}}
\end{aligned}
$$

and

$$
\begin{aligned}
& \zeta_{1}=\left(\frac{1}{2}+\frac{\Theta_{0}}{2 \sqrt{1+\Theta_{0}^{2}}}\right) \xi_{1}+\left(\frac{1}{2}-\frac{\Theta_{0}}{2 \sqrt{1+\Theta_{0}^{2}}}\right) \xi_{2}, \\
& \zeta_{2}=\left(\frac{1}{2}-\frac{\Theta_{0}}{2 \sqrt{1+\Theta_{0}^{2}}}\right) \xi_{1}+\left(\frac{1}{2}+\frac{\Theta_{0}}{2 \sqrt{1+\Theta_{0}^{2}}}\right) \xi_{2},
\end{aligned}
$$

where we denote $\Theta_{0}=\frac{\tilde{m}_{2}^{2}-\tilde{m}_{1}^{2}}{2 M_{12}^{2}}$.

As next step, we define

$$
\hat{\mathcal{H}}_{s}=\left(\begin{array}{cc}
H^{(1)} & 0 \\
0 & H^{(2)}
\end{array}\right)
$$

where $H^{(1)}=\left(\square-\Pi_{1}-\zeta_{1} R\right)$ and $H^{(2)}=\left(\square-\Pi_{2}-\zeta_{2} R\right)$, so that

$$
\operatorname{Tr} \ln \hat{\mathcal{H}}_{s}=\ln \operatorname{Det} \hat{\mathcal{H}}_{s}=\operatorname{Tr} \ln H^{(1)}+\operatorname{Tr} \ln H^{(2)} .
$$

Let us point out that using the rotation in the fields space to diagonalize $\hat{H}$, we also have to consider the contribution that comes from the transformation $\hat{\mathcal{H}}_{s}=U^{-1} \hat{H}_{S} U$. In relation (48), since $\operatorname{Det} U=\operatorname{Det} U^{-1}=1$, then there is no contribution. However the Jacobian of a such transformation in four-dimensional spacetime is proportional to $\delta^{4}(0)$, which in dimensional regularization formally vanishes while in our case via cut-off regularization scheme this means a cut-off dependent contribution to the cosmological constant.

Another important observation is that rotation (37) and an expansion to the first order in curvature are not commuting operations. This means that if we extract the $\mathcal{O}(R)$-term first and after that make a rotation only for a flat-space sector, the result would be different and not satisfactory from the point of view of our calculations.

Starting from this point, we meet a product of two normal scalar operators (47). In this situation it is possible to use the technique elaborated in [17] (see also $[4,18,20]$ ) to find the one-loop effective potential up to first order in $R$, using the Riemann normal coordinates formalism.

The equation for the propagator of a scalar field $G_{c}\left(x, x^{\prime}\right)$ related to $H^{(1)}$ has the form

$$
\left(g^{\frac{1}{4}} \square g^{-\frac{1}{4}}-\Pi_{1}-\zeta_{1} R\right) \bar{G}\left(x, x^{\prime}\right)=-\delta^{D}\left(x-x^{\prime}\right) .
$$

In Eq. (49) we take into account the expression for the covariant Dirac delta function

$\delta_{c}\left(x, x^{\prime}\right)=g^{-1 / 4} \delta^{D}\left(x-x^{\prime}\right) g^{\prime-1 / 4}$

and the modified propagator $\bar{G}\left(x, x^{\prime}\right)$ [17]. Both elements are necessary for the consistency of the expansion, so that the r.h.s. of the above equation does not depend on the metric tensor. Thus, one can use the relation $\operatorname{Tr} \ln \hat{H}=$ - $\operatorname{Tr} \ln \bar{G}$ to derive the dependence on the curvature tensor in Eq. (25).

In the Riemann normal coordinates the expansion of the spacetime metric $g_{\alpha \beta}$ up to first order in the curvature is given by [21] (see also simplified introduction and more references in [22])

$g_{\alpha \beta}(x)=\eta_{\alpha \beta}-\frac{1}{3} R_{\alpha \mu \beta \nu}\left(x^{\prime}\right) y^{\mu} y^{v}+\cdots$,

hence

$$
\begin{aligned}
R(x)= & R\left(x^{\prime}\right)+\cdots, \\
\square= & \partial^{2}+\frac{1}{3} R_{\alpha \beta}^{\mu \nu}\left(x^{\prime}\right) y^{\alpha} y^{\beta} \partial_{\mu} \partial_{v}-\frac{2}{3} R_{\beta}^{\alpha}\left(x^{\prime}\right) y^{\beta} \partial_{\alpha} \\
& +\cdots .
\end{aligned}
$$


Starting from this formula, it is easy to get

$$
\begin{aligned}
g^{1 / 4} \square g^{-1 / 4}= & \partial^{2}+\frac{1}{6} R+\frac{1}{3}\left(R_{\alpha \beta}^{\mu \nu}\left(x^{\prime}\right) y^{\alpha} y^{\beta} \partial_{\mu} \partial_{\nu}\right. \\
& \left.-R_{\beta}^{\alpha}\left(x^{\prime}\right) y^{\beta} \partial_{\alpha}\right)+\cdots,
\end{aligned}
$$

where the derivatives are $\partial_{\alpha}=\frac{\partial}{\partial y^{\alpha}}, \partial^{2}=\eta^{\mu \nu} \partial_{\mu} \partial_{\nu}$ and the dots mean higher order terms in the curvature tensor and its covariant derivatives.

After all, Eq. (49) becomes

$\left[-\partial^{2}+\Pi_{1}+\left(\zeta_{1}-\frac{1}{6}\right) R\right] \bar{G}\left(x, x^{\prime}\right)=\delta^{D}\left(x-x^{\prime}\right)$.

We can also note that the last term in Eq. (54) does not contribute to the effective potential due to the Lorentz invariance [17].

The solution up to the first order in the curvature has the form

$$
\begin{aligned}
\bar{G}\left(x, x^{\prime}\right)= & \int \frac{d^{D} k}{(2 \pi)^{D}} e^{i k y}\left[\frac{1}{k^{2}+\Pi_{1}}\right. \\
& \left.-\left(\zeta_{1}-\frac{1}{6}\right) \frac{R}{\left(k^{2}+\Pi_{1}\right)^{2}}\right] .
\end{aligned}
$$

The results presented above, enable one to find the oneloop effective potential. Taking into account the expansion of bilinear operator $H^{(1)}$ and the Green's function $\bar{G}\left(x, x^{\prime}\right)$ up to first order in $R$, we have

$$
\begin{aligned}
-\operatorname{Tr} \ln \bar{G} & =\operatorname{Tr} \ln \left(\hat{H}_{0}^{(1)}+\hat{H}_{1}^{(1)} R\right) \\
& =\operatorname{Tr} \ln \hat{H}_{0}^{(1)}+\operatorname{Tr} \bar{G}_{0} \hat{H}_{1}^{(1)} R .
\end{aligned}
$$

For the effective potential in flat spacetime we need just the first term in the r.h.s. of Eq. (57) given by

$$
\begin{aligned}
-\int d^{D} x \bar{V}_{0}^{(1)}= & \frac{1}{2} \operatorname{Tr} \ln \hat{H}_{0}^{(1)} \\
= & \frac{1}{2} \operatorname{Tr} \ln S_{2}(\varphi, \chi) \\
& -\frac{1}{2} \operatorname{Tr} \ln S_{2}(\varphi=\chi=0),
\end{aligned}
$$

where $S_{2}(\phi)$ is the bilinear form of the classical action in the background-field formalism. The last term in Eq. (58) can be seen as a normalization of the functional integral. This term arises naturally through the diagrammatic representation of effective potential. From Eq. (58) we get

$\bar{V}_{0}^{(1)}(\varphi, \chi)=\frac{1}{2} \int \frac{d^{D} k}{(2 \pi)^{D}} \ln \left(\frac{k^{2}+\Pi_{1}}{k^{2}+m_{1}^{2}}\right)$.

Using the Euclidean momentum cut-off $\Omega$, for $D=4$ we have

$\bar{V}_{0}^{(1)}(\varphi, \chi)=\frac{1}{2(4 \pi)^{2}} \int_{0}^{\Omega} d k^{2} k^{2} \ln \left(\frac{k^{2}+\Pi_{1}}{k^{2}+m_{1}^{2}}\right)$, and we finally get

$$
\bar{V}_{0}^{(1)}(\varphi, \chi)=\bar{V}_{0-d i v}^{(1)}(\varphi, \chi)+\bar{V}_{0-f i n}^{(1)}(\varphi, \chi),
$$

where

$$
\begin{aligned}
\bar{V}_{0-d i v}^{(1)}(\varphi, \chi)= & \frac{1}{32 \pi^{2}}\left\{\Omega^{2}\left(\Pi_{1}-m_{1}^{2}\right)-\frac{\Pi_{1}^{2}}{2} \ln \frac{\Omega^{2}}{\mu^{2}}\right. \\
& \left.+\frac{1}{2} m_{1}^{4} \ln \frac{\Omega^{2}}{\mu^{2}}\right\}, \\
\bar{V}_{0-f i n}^{(1)}(\varphi, \chi)= & \frac{1}{32 \pi^{2}}\left\{-\frac{1}{4}\left(\Pi_{1}^{2}-m_{1}^{4}\right)+\frac{\Pi_{1}^{2}}{2} \ln \frac{\Pi_{1}}{\mu^{2}}\right. \\
& \left.-\frac{1}{2} m_{1}^{4} \ln \frac{m_{1}^{2}}{\mu^{2}}\right\} .
\end{aligned}
$$

The second term in the r.h.s. of Eq. (57) corresponds to the first order in curvature correction $\bar{V}_{1}^{(1)}(\varphi, \chi)$, which can be derived as follows

$$
\begin{aligned}
& -\int d^{D} x \bar{V}_{1}^{(1)}=\frac{1}{2} \operatorname{Tr} \bar{G}_{0} \hat{H}_{1} R \\
& =-\frac{1}{2} \int d^{D} x \int d^{D} x^{\prime} \bar{G}_{0}^{-1}\left(x, x^{\prime}\right) \bar{G}_{1}\left(x^{\prime}, x\right) R,
\end{aligned}
$$

so that

$$
\begin{aligned}
\bar{V}_{1}^{(1)}= & \frac{1}{2} \int d^{D} x^{\prime} \int \frac{d^{D} k}{(2 \pi)^{D}} e^{i k\left(x-x^{\prime}\right)} \\
& \times \int \frac{d^{D} p}{(2 \pi)^{D}} e^{i p\left(x^{\prime}-x\right)} \bar{G}_{0}^{-1}(k) \bar{G}_{1}(p) R \\
= & \frac{1}{2} \int \frac{d^{D} k}{(2 \pi)^{D}} \bar{G}_{0}^{-1}(k) \bar{G}_{1}(k) R .
\end{aligned}
$$

For $D=4$ and replacing the explicit forms of $\bar{G}_{0}^{-1}(k)$ and $\bar{G}_{1}(k)$ of Eq. (56) in Eq. (65), one arrives at

$$
\bar{V}_{1}^{(1)}=-\frac{1}{2(2 \pi)^{4}}\left(\zeta_{1}-\frac{1}{6}\right) R \int_{0}^{\Omega} \frac{k^{2} d k^{2}}{k^{2}+\Pi_{1}} .
$$

After taking the last integral, the result has the form

$\bar{V}_{1}^{(1)}(\varphi, \chi)=\bar{V}_{1-d i v}^{(1)}(\varphi, \chi)+\bar{V}_{1-f i n}^{(1)}(\varphi, \chi)$,

where

$$
\begin{aligned}
& \bar{V}_{1-d i v}^{(1)}(\varphi, \chi)=-\frac{1}{32 \pi^{2}}\left(\zeta_{1}-\frac{1}{6}\right) R\left[\Omega^{2}-\Pi_{1} \ln \frac{\Omega^{2}}{\mu^{2}}\right] \\
& \bar{V}_{1-f i n}^{(1)}(\varphi, \chi)=-\frac{1}{32 \pi^{2}}\left(\zeta_{1}-\frac{1}{6}\right) R \Pi_{1} \ln \frac{\Pi_{1}}{\mu^{2}}
\end{aligned}
$$

We have described the calculations for the first contribution due to $\hat{H}^{(1)}$. For the second term $\hat{H}^{(2)}$ the calculations are analogous except that in this case we have to use $\Pi_{2}$ and $\zeta_{2}$ instead $\Pi_{1}$ and $\zeta_{1}$. The final result has the form 


$$
\begin{aligned}
\bar{V}(\varphi, \chi)= & \bar{V}_{0}^{(1)}(\varphi, \chi)+\bar{V}_{0}^{(2)}(\varphi, \chi) \\
& +\bar{V}_{1}^{(1)}(\varphi, \chi)+\bar{V}_{1}^{(2)}(\varphi, \chi) \\
= & \frac{1}{32 \pi^{2}}\left\{-\frac{1}{4}\left[\Pi_{1}^{2}+\Pi_{2}^{2}-\left(m_{1}^{4}+m_{2}^{4}\right)\right]\right. \\
& +\Omega^{2}\left[\Pi_{1}+\Pi_{2}-\left(m_{1}^{2}+m_{2}^{2}\right)\right] \\
& +\frac{1}{2}\left(\Pi_{1}^{2}+\Pi_{2}^{2}\right) \ln \frac{\Pi_{2}}{\mu^{2}}-\frac{1}{2}\left(\Pi_{1}^{2}+\Pi_{2}^{2}\right) \ln \frac{\Omega^{2}}{\mu^{2}} \\
& -\frac{1}{2} m_{1}^{4} \ln \frac{m_{1}^{2}}{\mu^{2}}-\frac{1}{2} m_{2}^{4} \ln \frac{m_{2}^{2}}{\mu^{2}} \\
& +\frac{1}{2}\left(m_{1}^{4}+m_{2}^{4}\right) \ln \frac{\Omega^{2}}{\mu^{2}} \\
& -\left(\zeta_{1}-\frac{1}{6}\right) R\left[\Pi_{1} \ln \frac{\Pi_{1}}{\mu^{2}}+\Omega^{2}-\Pi_{1} \ln \frac{\Omega^{2}}{\mu^{2}}\right] \\
& \left.-\left(\zeta_{2}-\frac{1}{6}\right) R\left[\Pi_{2} \ln \frac{\Pi_{2}}{\mu^{2}}+\Omega^{2}-\Pi_{2} \ln \frac{\Omega^{2}}{\mu^{2}}\right]\right\} .
\end{aligned}
$$

This is the final result for scalar fields loop to effective potential.

Some observations concerning the expression (69) are in order. First of all, the divergent part is in the perfect correspondence with the corresponding part of the result (15), obtained on the base of the heat-kernel method. In order to see this it is sufficient to use the well-known correspondence between covariant cut-off and dimensional regularization parameter (see, e.g., [23]),

$\frac{2}{4-n} \mu^{n-4} \sim \ln \frac{\Omega^{2}}{\mu^{2}}, \quad n \longrightarrow 4$.

Second, the dependence on the renormalization parameter $\mu$ is exactly the standard one, such that the effective potential is a solution of the standard renormalization group equation (27).

Thus, the expression (69) indicates that the quantum corrections are given by some logarithmic terms, similar to the general renormalization group-based form (28). On the other hand, the logarithmic terms in (69) depend on the unusual arguments representing the mixture of different scalar fields, their masses and coupling constants. This situation is in fact typical for the quantum corrections coming from the loops with mixed internal lines, e.g. of the light and heavy mass fields [11] (see also a recent work [12] for the extension to curved space). However, it is interesting to point out that this form of the effective potential does not confirm a naive expectation that the scalar fields contribution to effective potential can be obtained using the anzatz of the form $t^{(0)}$ from Eq. (29) for each of the background scalars. This output means that the possibility to derive the full result (69) from the renormalization group equation is not evident and deserves a further study.
We can point out that in the limit of large scalar fields, when both $|\varphi| \rightarrow \infty$ and $|\chi| \rightarrow \infty$, our result (69) reduce to the sum of logarithmic contributions of the scalar fields. However, in general the effective potential has more complicated form. The origin of this feature of the two-scalar model is the rotation (37), that mixes different masses, interactions and non-minimal parameters.

\section{Conclusions and perspectives}

We have formulated the Yukawa model of one sterile scalar and one axial scalar (pseudoscalar) fields, interacting to themselves and also to the set of fermions through the Yukawa couplings.

The power counting analysis of the divergences shows that the helps us to identify the form of the classical potential of scalar and pseudoscalar self-interaction, providing renormalizable quantum theory. This potential has all even and odd terms that are allowed by symmetries (including parity) of the classical theory, without coupling constants with the inverse-mass dimensions.

The complete analysis of one-loop renormalization, $\beta-$ and $\gamma$-functions was given in Sect. 3. The main results of this part is the importance of the mixed scalar-pseudoscalar terms, which do not have symmetry protection and, as a result, are indispensable for renormalizability of the theory. Thus, we have completely described the one-loop renormalization structure of the model under consideration.

The effective potential has been calculated up to the linear in scalar curvature terms. The results is a sum of independent contributions from the scalar fields loop and from the spinor field loop. The contributions of the scalar sector has been calculated in the explicit form and demonstrate a nontrivial dependence on the background scalar fields, on masses and coupling constants. Let us note that the derivation of the fermion contribution to effective potential in the full massive theory faces serious technical difficulties and we left it for the future work.

It is interesting that unlike the single scalar field models, the effective potential in the two-scalar model under discussion contains usual logarithmic terms and also the terms with the non-logarithmic asymptotic. In the scalar loop sector the model under consideration is qualitatively similar to the situation with two quantum fields with different masses, that is well-known from the literature (see, e.g. [11]) and was recently discussed in curved space [12]. It is remarkable, however, that in our expressions we could observe the effect of masses even in the local effective potential, without invoking the non-local form factors, as it is done in the mentioned publications. It is worth mentioning, that the direct calculation of the scalar loop for effective potential has been performed using rotation (37) in the space of the scalar fields. 
This operation turns out to be not commutative with the expansion using local momentum representation.

Acknowledgements E.A.R. is grateful to Coordenação de Aperfeiçoamento de Pessoal de Nível Superior - CAPES for supporting his Ph.D. project. I.B. is grateful to CAPES for supporting his long-term visit to UFJF and to the Physics Departament of UFJF for kind hospitality during the period when this work started. Also he thanks the Russian Ministry of Science and High Education, project no. 3.1386.2017 for partial support. The work of I.L.Sh. was partially supported by Conselho Nacional de Desenvolvimento Científico e Tecnológico - CNPq under the grant 303635/2018-5 and Fundação de Amparo à Pesquisa de Minas Gerais - FAPEMIG under the project APQ-01205-16.

Data Availability Statement This manuscript has associated data in a data repository. [Author's comment: The datasets generated during and/or analysed during the current study are available from the corresponding author on reasonable request.]

Open Access This article is distributed under the terms of the Creative Commons Attribution 4.0 International License (http://creativecomm ons.org/licenses/by/4.0/), which permits unrestricted use, distribution, and reproduction in any medium, provided you give appropriate credit to the original author(s) and the source, provide a link to the Creative Commons license, and indicate if changes were made.

Funded by SCOAP ${ }^{3}$.

\section{Appendix}

The intermediate expressions leading to (15) are

$$
\begin{aligned}
& \nabla_{\mu} \hat{h}^{\mu}=\left(\begin{array}{ccc}
0 & 0 & \frac{i}{2} h_{1} \nabla_{\mu} \bar{\Psi}_{j} \gamma^{\mu} \\
0 & 0 & \frac{i}{2} h_{2} \nabla_{\mu} \bar{\Psi}_{j} \gamma^{5} \gamma^{\mu} \\
0 & 0 & \frac{i}{2}\left(h_{1} \nabla_{\mu} \varphi+h_{2} \nabla_{\mu} \chi \gamma^{5}\right) \gamma^{\mu} \delta^{i j}
\end{array}\right), \\
& \hat{h}_{\mu} \hat{h}^{\mu}=\left(\begin{array}{ccc}
0 & 0 & -h_{1}^{2} \bar{\Psi}_{k} \varphi+h_{1} h_{2} \bar{\Psi}_{k} \chi \gamma^{5} \\
0 & 0 & -h_{1} h_{2} \bar{\Psi}_{k} \gamma^{5} \varphi+h_{2}^{2} \bar{\Psi}_{k} \chi \\
0 & 0 & \left(-h_{1}^{2} \varphi^{2}+h_{2}^{2} \chi^{2}\right) \delta^{i k}
\end{array}\right)
\end{aligned}
$$

and

$$
\hat{h}_{\mu} \hat{h}_{v}=\left(\begin{array}{ccc}
0 & 0 & -\frac{1}{4}\left(h_{1}^{2} \bar{\Psi}_{k} \varphi-h_{1} h_{2} \bar{\Psi}_{k} \chi \gamma^{5}\right) \gamma_{\mu} \gamma_{\nu} \delta^{i k} \\
0 & 0 & -\frac{1}{4}\left(h_{1} h_{2} \bar{\Psi}_{k} \varphi \gamma^{5}-h_{2}^{2} \bar{\Psi}_{k} \chi\right) \gamma_{\mu} \gamma_{\nu} \delta^{i k} \\
0 & 0 & -\frac{1}{4}\left(h_{1}^{2} \varphi^{2}-h_{2}^{2} \chi^{2}\right) \gamma_{\mu} \gamma_{\nu}
\end{array}\right) .
$$

As a result, the elements of the matrices $\hat{P}$ and $\hat{S}_{\mu \nu}$ in Eq. (12) have the form

$$
\begin{aligned}
& P_{11}=m_{1}^{2}+\frac{\lambda_{1} \varphi^{2}}{2}+g \varphi-\left(\xi_{1}-\frac{1}{6}\right) R+\lambda_{3} \chi^{2}, \\
& P_{12}=2 p \chi+4 \lambda_{3} \varphi \chi, \quad P_{21}=2 p \chi+4 \lambda_{3} \varphi \chi, \\
& P_{13}=h_{1} \bar{\Psi}_{k}\left(M+h_{1} \varphi-h_{2} \chi \gamma^{5}\right)-\frac{i}{2} h_{1}\left(\nabla_{\mu} \bar{\Psi}_{k}\right) \gamma^{\mu}, \\
& P_{22}=m_{2}^{2}+\frac{\lambda_{2} \chi^{2}}{2}+p \varphi-\left(\xi_{2}-\frac{1}{6}\right) R+\lambda_{3} \varphi^{2},
\end{aligned}
$$

$$
\begin{aligned}
P_{23}= & h_{2} \bar{\Psi}_{k}\left(M \gamma^{5}+h_{1} \varphi \gamma^{5}-h_{2} \chi\right)-\frac{i}{2} h_{2}\left(\nabla_{\mu} \bar{\Psi}_{k}\right) \gamma^{5} \gamma^{\mu} \\
P_{31}= & 2 h_{1} \Psi_{i}, \quad P_{32}=2 h_{2} \gamma^{5} \Psi_{i} \\
P_{33}= & {\left[M^{2}-\frac{1}{12} R+h_{1} M \varphi+h_{2} M \chi \gamma^{5}+h_{1}^{2} \varphi^{2}-h_{2}^{2} \chi^{2}\right.} \\
& \left.-\frac{i}{2}\left(h_{1} \nabla_{\mu} \varphi+h_{2} \nabla_{\mu} \chi \gamma^{5}\right) \gamma^{\mu}\right] \delta^{i k}
\end{aligned}
$$

and

$$
\begin{aligned}
S_{\mu \nu 13}= & -\frac{i}{2} h_{1}\left[\left(\nabla_{\mu} \bar{\Psi}_{k}\right) \gamma_{\nu}-\left(\nabla_{\nu} \bar{\Psi}_{k}\right) \gamma_{\mu}\right] \\
& +\frac{1}{4}\left(h_{1}^{2} \bar{\Psi}_{k} \varphi-h_{1} h_{2} \bar{\Psi}_{k} \chi \gamma^{5}\right)\left[\gamma_{\mu}, \gamma_{\nu}\right], \\
S_{\mu \nu 23}= & -\frac{i}{2} h_{2}\left[\left(\nabla_{\mu} \bar{\Psi}_{k}\right) \gamma^{5} \gamma_{\nu}-\left(\nabla_{\nu} \bar{\Psi}_{k}\right) \gamma^{5} \gamma_{\mu}\right] \\
& +\frac{1}{4}\left(h_{1} h_{2} \varphi \bar{\Psi}_{k} \gamma^{5}-h_{2}^{2} \bar{\Psi}_{k} \chi\right)\left[\gamma_{\mu}, \gamma_{\nu}\right], \\
S_{\mu \nu 33}= & {\left[\nabla_{\nu}, \nabla_{\mu}\right]-\frac{i}{2} h_{1}\left[\left(\nabla_{\mu} \varphi\right) \gamma_{\nu}-\left(\nabla_{\nu} \varphi\right) \gamma_{\mu}\right] } \\
& -\frac{i}{2} h_{2}\left[\left(\nabla_{\mu} \chi\right) \gamma^{5} \gamma_{\nu}-\left(\nabla_{\nu} \chi\right) \gamma^{5} \gamma_{\mu}\right] \\
& \left.+\frac{1}{4}\left(h_{1}^{2} \varphi^{2}-h_{2}^{2} \chi^{2}\right)\left[\gamma_{\mu}, \gamma_{\nu}\right]\right] \delta^{i k} .
\end{aligned}
$$

\section{References}

1. D.J. Toms, JHEP 1805, 139 (2018). arXiv: 1804.08350

2. D.J. Toms, Phys. Rev. D 98, 025015 (2018). arXiv: 1805.01700

3. D.J. Toms. arXiv: 1906.02515

4. V.F. Barra, I.L. Buchbinder, J.G. Joaquim, A.R. Rodrigues, I.L. Shapiro, Eur. Phys. J. C 79, 458 (2019). arXiv:1903.11546

5. I.L. Buchbinder, I.L. Shapiro, Class. Quantum Gravity 7, 1197 (1990)

6. I.L. Buchbinder, S.D. Odintsov, I.L. Shapiro, Effective Action in Quantum Gravity (IOP Publishing, Bristol, 1992)

7. S.R. Coleman, E.J. Weinberg, Phys. Rev. D 7, 1888 (1973)

8. I.L. Buchbinder, S.D. Odintsov, Sov. Phys. J. 27, 554 (1984)

9. I.L. Buchbinder, J.J. Wolfengaut, Class. Quantum Gravity 5, 1127 (1988)

10. S. Alexander, N. Yunes, Phys. Rep. 480, 1 (2009). arXiv:0907.2562

11. V. Ilisie, Concepts in Quantum Field Theory. A Practitioner's Toolkit (Springer, New York, 2016)

12. T.G. Ribeiro, I.L. Shapiro, arXiv: 1908.01937

13. N.D. Birell, P.C.W. Davies, Quantum Fields in Curved Space (Cambridge University Press, Cambridge, 1982)

14. B.S. DeWitt, Dynamical Theory of Groups and Fields (Gordon and Breach, New York, 1965)

15. I.L. Buchbinder, Theor. Math. Phys. 61, 1215 (1984)

16. D.J. Toms, Phys. Lett. B 126, 37 (1983)

17. T.S. Bunch, L. Parker, Phys. Rev. D 20, 2499 (1979)

18. F. Sobreira, B.J. Ribeiro, I.L. Shapiro, Phys. Lett. B 705, 273 (2011)

19. S.-B. Liao, Phys. Rev. D 53, 2020 (1996)

20. E.A. Reis, G. Krein, Td P. Netto, I.L. Shapiro, Phys. Lett. B 798, 134925 (2019). arXiv:1804.04569

21. A.Z. Petrov, Einsten Spaces (Pergamon Press, Oxford, 1969)

22. I.L. Shapiro, Primer in Tensor Analysis and Relativity (Springer, New York, 2019)

23. A.O. Barvinsky, G.A. Vilkovisky, Phys. Rep. 119, 1 (1985) 